\title{
Initial experience with percutaneous endoscopic gastrostomy with T-fastener fixation in pediatric patients
}

\section{다(1)(우}

\author{
Authors \\ Ragnhild Emblem ${ }^{1,2}$, Kristin Bjørnland ${ }^{1,2}$ \\ Institutions \\ 1 Institute of Clinical Medicine, University of Oslo, Oslo, \\ Norway \\ 2 Department of Gastrointestinal and Pediatric Surgery, \\ Oslo University Hospital, Oslo, Norway \\ 3 Department of Pediatrics, Oslo University Hospital, \\ Oslo, Norway
}

Morten Kvello ${ }^{1,2}$, Charlotte Kristensen Knatten ${ }^{3}$, Gøri Perminow ${ }^{3}$, Hans Skari², Anders Engebretsen², Ole Schistad²,

submitted 25.4 .2017

accepted after revision 20.9.2017

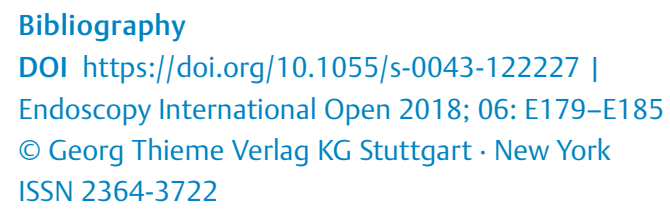

Corresponding author

Morten Kvello, Department of Gastrointestinal and Pediatric Surgery, Oslo University Hospital, Rikshospitalet, Postboks 4950 Nydalen, 0424 Oslo, Norway

Fax: +47-2-3072526

m.kvello@gmail.com

\section{ABSTRACT}

Background and study aims Insertion of a percutaneous endoscopic gastrostomy (PEG) with push-through technique and T-fastener fixation (PEG-T) has recently been introduced in pediatric patients. The T-fasteners allow a pri- mary insertion of a balloon gastrostomy. Due to limited data on the results of this technique in children, we have investigated peri- and postoperative outcomes after implementation of PEG-T in our department.

Patients and methods This retrospective chart review included all patients below 18 years who underwent PEG-T placement from 2010 to 2014. Main outcomes were 30day postoperative complications and late gastrostomyrelated complications.

Results In total, 87 patients were included, and median follow-up time was 2.4 years ( 1 month -4.9 years). Median age and weight at PEG-T insertion were 1.9 years (9.4 months - 16.4 years) and $10.4 \mathrm{~kg}(5.4-33.0 \mathrm{~kg})$, respectively. Median operation time was 28 minutes (1065 minutes), and 6 surgeons and 3 endoscopists performed the procedures. During the first 30 days, 54 complications occurred in 41 patients (47\%). Most common were peristomal infections treated with either local antibiotics in 11 patients $(13 \%)$ or systemic antibiotics in 11 other patients (13\%). 9 patients (10\%) experienced tube dislodgment. Late gastrostomy-related complications occurred in 33 patients (38\%). The T-fasteners caused early and late complications in $9(10 \%)$ and 11 patients (13\%), respectively. Of these, 4 patients ( $5 \%$ ) had subcutaneously migrated T-fasteners which were removed under general anesthesia.

Conclusion We found a high rate of complications after PEG-T. In particular, problems with the T-fasteners and tube dislodgment occurred frequently after PEG-T insertion.

\section{Introduction}

The standard management of pediatric patients requiring longterm enteral nutritional support is a gastrostomy feeding tube [1]. Most centers prefer to insert a percutaneous endoscopic gastrostomy (PEG) owing to its simplicity and low costs [2]. The pull technique (PEG-P) is the most commonly used method, and an internal bumper secures fixation of the stomach to the abdominal wall. In adults, removal of the PEG-P tube is usually done with the "cut and push" technique. This is not re- commended in children due to reports of severe complications such as esophageal laceration and intestinal obstruction caused by the internal bumper [3]. Therefore, in pediatric patients, the tube is removed either endoscopically or by traction, under general anesthesia or sedation [4].

An alternative to PEG-P is the push technique, which offers primary insertion of a balloon gastrostomy tube or a low-profile button. In pediatric patients, this is a major advantage as the tube can easily be removed without the need for sedation or general anesthesia. Since the balloon does not offer the same 



- Fig. 1 Technique for placement of a percutaneous endoscopic gastrostomy with T-fastener gastropexy (PEG-T). a Gastropexy is performed by placing $3 \mathrm{~T}$-fasteners with resorbable sutures in a trigonal fashion. $\mathbf{b}$ Using a modified Seldinger technique, serial dilators are used to establish the stoma channel. c A balloon-retained device is then introduced, and the balloon filled with sterile water. $\mathbf{d}$ A low profile button in place with surrounding external suture locks.

secure fixation of the stomach to the abdominal wall as an internal bumper, the stomach must be fixed to the abdominal wall by sutures or T-fasteners. Another postulated benefit of the push technique is decreased risk for peristomal infections because the tube is not exposed to the oral bacterial flora during insertion. Although the push technique is associated with a lower peristomal infection rate than PEG-P in adults [5], a similar advantage has not been shown in pediatric patients [6, 7].

Recently, PEG placement with the push technique and T-fastener gastropexy (PEG-T) has been introduced in pediatric patients [6], and this particular method has gained popularity. So far, there are only a few studies that have evaluated the PEG-T technique in pediatric patients [6-8], therefore, we wanted to report our initial experience with the PEG-T with particular emphasis on peri- and postoperative complications.

\section{Materials and methods}

\section{Patients}

This retrospective, descriptive study includes all patients $<18$ years of age who received a PEG-T at Oslo University Hospital Ullevål between January 2010 and November 2014. PEG-T was introduced at our center in January 2010 and was the preferred technique for PEG if no contraindication was found. Patients receiving PEG-T were identified by an electronic search for the PEG procedure and cross-checked with data from a written surgical logbook.

\section{Technique for PEG-T placement}

The MIC-KEY Introducer Kit (Kimberly-Clark Corp, Roswell, GA, United States) was used in all patients, and the gastrostomy was placed according to the manufacturer's procedure as previously described by Göthberg and Björnsson [6]. The standard tube was a $14 \mathrm{Fr}$ bolus gastrostomy balloon tube. A low-profile button was placed initially in some patients according to parents' wish or if assessed appropriate by the gastroenterologist. All procedures were performed under general anesthesia as well as local anesthesia around the stoma channel. Prophylactic antibiotic (Cefalotin) was given before the procedure. Briefly, the stomach was distended by insufflation, and a suitable location for the gastrostomy tube and surrounding gastropexy points was identified by transillumination. Gastropexy was performed using $3 \mathrm{~T}$-fasteners with resorbable sutures (Saf-T-Pexy T-fasteners, Kimberly-Clark Corp). These were placed in a trigonal fashion and fastened with accompanying external suture locks. A stoma channel for the gastrostomy tube was established in the center of the trigone of T-fasteners using a modified Seldinger technique and a serial $18 \mathrm{Fr}$ dilatator. Lastly, the $14 \mathrm{Fr}$ gastrostomy tube was inserted, and the balloon filled with sterile water ( $\triangleright$ Fig. $\mathbf{1}$ ).

Before the introduction of the PEG-T procedure, the technique was presented to the team by a representative from the company, and an introduction CD showing the technique was available for the team.

\section{Postoperative treatment}

Enteral liquids were started 6 hours after the procedure, and tube feeding started on the first postoperative day. Patients were discharged to their home or to their local hospital as appropriate. The parents or a gastrostomy nurse were instructed to remove the suture locks 3-4 weeks postoperatively if these had not already detached spontaneously. The balloon tube was scheduled to be replaced with a low profile button at our hospital or at the patient's local hospital 8 weeks after surgery if a button was not inserted primarily.

\section{Collection of data}

Patient data were collected from electronic medical records. Demographic data included age, sex, weight, presence of a nasogastric tube at admittance, and underlying diagnosis most likely contributing to the patients' feeding problems. Neurologic impairment was defined as a static or progressive, central or peripheral neurologic condition associated with chronic functional or intellectual impairment [9], including conditions such as cerebral palsy, quadriplegia, and genetic syndromes. Duration of the procedure, perioperative complications, and any concomitant procedure were recorded. Operating time was not registered in patients undergoing concomitant procedures. 
Postoperative complications were graded according to the Clavien-Dindo classification for surgical complications [10]. Briefly, grade I complications indicate any deviation from the normal postoperative course, but without the need for any pharmacologic treatment. Grade II complications require pharmacologic treatment with drugs. Grade III complications require surgical, endoscopic, or radiologic intervention. Interventions not requiring or requiring general anesthesia were denoted as grade IIIa and IIIb, respectively. Grade IV is life-threatening complications, whereas grade $V$ describes death of a patient.

Postoperative complications were categorized as early ( $\leq 30$ days) or late (>30 days). Early complications included all types of complications, but non-gastrostomy related complications (e.g. pneumonia) were excluded if concomitant procedures were performed. Late complications were limited to gastrostomy related complications only. Stoma infections were categorized as grade I if treated with topical agents or as grade II if systemic antibiotics were given. Complications specifically related to the T-fasteners were registered as a separate item with attention to when and how they were discovered and when and how they were treated.

\section{Ethical approval}

The study has been approved by the hospital's Commission for Personal Security.

\section{Statistics}

Descriptive statistics were used when presenting the data; frequencies and percentages, median and range [min-max]. Median operating time in the first and last half of patients was compared using a Mann-Whitney $U$ test, as data were not normally distributed. The frequency of early postoperative complications in various subgroups was compared using the chisquared test. All analyses were performed by IBM SPSS software for Windows version 21.0 (IBM Corp, Armonk, NY, United States). $P$ values $<0.05$ were considered statistically significant.

\section{Results}

\section{Patients}

A total of 87 patients were identified, of whom 45 (52\%) were female. Median age and weight were 1.9 years (2.9 months 16.4 years) and $10.4 \mathrm{~kg}(5.4-33.0 \mathrm{~kg})$, respectively. The most common underlying diagnosis was neurologic impairment $(n=$ $53,61 \%$ ), followed by weight faltering of unknown etiology $(n=$ $13,15 \%)$, malignancy $(n=3,3 \%)$, and other diseases $(n=18$, $21 \%$ ). A nasogastric tube was present in 33 patients (38\%) at admittance. The median follow-up time was 2.3 years ( 1 month -4.8 years).

\section{Perioperative data}

Three consultant pediatric gastroenterologists and 6 pediatric surgeons ( 5 consultants or 1 trainee) performed the PEG-T procedures during the study period. $6(7 \%)$ patients underwent a concomitant procedure; central venous catheter insertion ( $\mathrm{n}=$ $2)$, tooth extraction $(n=1)$, eye examination $(n=1)$, broncho-
- Table 1 Early postoperative complications in pediatric patients undergoing insertion of a percutaneous endoscopic gastrostomy with Tfastener gastropexy.

\begin{tabular}{|c|c|}
\hline & $\begin{array}{l}\text { Early complications } \\
(n=54)\end{array}$ \\
\hline Grade I & 27 \\
\hline - Stoma infection & 11 \\
\hline - Tube dislodgment ${ }^{1}$ & 4 \\
\hline - Granulation tissue & 3 \\
\hline - Minor bleeding & 3 \\
\hline - Leakage & 1 \\
\hline - Aspiration pneumonia & 1 \\
\hline - Detached external suture lock & 1 \\
\hline - Others ${ }^{2}$ & 3 \\
\hline Grade II & 21 \\
\hline - Stoma infection & 11 \\
\hline - Pneumonia & 4 \\
\hline - Serious feeding difficulties & 2 \\
\hline - Tube dislodgment ${ }^{3}$ & 1 \\
\hline - Pain from external suture lock & 1 \\
\hline - Others ${ }^{2}$ & 2 \\
\hline Grade IIIb & 6 \\
\hline - Tube dislodgment & 4 \\
\hline - Leakage & 1 \\
\hline - Stoma infection & 1 \\
\hline \multicolumn{2}{|c|}{$\begin{array}{l}{ }^{1} \text { Bedside reintroduction of the tube. } \\
\left.{ }^{2} \text { Others include for grade I: pneumoperitoneum ( } n=1\right) \text {, gastroenteritis } \\
(n=1) \text {, urinary retention }(n=1) \text {, and for grade II: fungal infection around a } \\
\text { central venous catheter }(n=1) \text {, laryngitis }(n=1) \text {. } \\
{ }^{3} \text { Tube reinserted under Midazolam anesthesia. }\end{array}$} \\
\hline
\end{tabular}

scopy $(n=1)$, and muscle biopsy $(n=1)$. The median duration of the PEG-T procedure in the remaining 81 patients was 28 minutes ( $10-65$ minutes). In the first 40 patients, the median operating time was 28 minutes compared to 27 minutes in the last 41 patients $(P=0.26)$.

In 7 patients, 1 or more of the T-fasteners detached or was placed at an unsuitable location and these were therefore replaced by a new T-fastener during the PEG procedure. There were no other perioperative complications.

\section{Early postoperative complications}

A total of 54 early postoperative complications were recorded in $41(47 \%)$ patients ( $\triangleright$ Table 1 ) of which the majority were grade I and II complications. Of these, 45 were gastrostomy related. Of the patients with an early stoma infection treated with topical agents (grade I) or systemic antibiotics (grade II), 5 and 3 patients, respectively, also had an infection around the external suture locks. Of the 6 patients (7\%) with early grade 
- Table 2 Early postoperative complications following insertion of a percutaneous endoscopic gastrostomy with T-fasteners (PEG-T) needing treatment under general anesthesia (grade IIIb complications).

\begin{tabular}{|l|l|l|l|l|}
\hline $\begin{array}{l}\text { Patient } \\
\text { number }\end{array}$ & Clinical findings & $\begin{array}{l}\text { Treatment } \\
\text { modality }\end{array}$ & $\begin{array}{l}\text { Time to } \\
\text { reoperation }\end{array}$ & Intraoperative findings \\
\hline 1 & Tube dislodgment, clinical sign of peritonitis & Laparotomy & 4 days & No peritoneal leakage, no organ injury \\
\hline 2 & Tube dislodgment & Laparotomy & 1 day & No injury \\
\hline 3 & Infection, pneumoperitoneum & Laparotomy & 7 days & No organ injury, wide stoma channel \\
\hline 4 & Feces-like leakage from stoma channel & Laparotomy & 2 days & No organ injury, wide stoma channel \\
\hline 5 & Tube dislodgment & Endoscopy & Same day & T-fasteners in place \\
\hline 6 & Tube dislodgment & Endoscopy & Same day & T-fasteners in place \\
\hline${ }^{1}$ Laparotomy was performed because transillumination was suboptimal during the initial PEG-T insertion. & \\
\hline
\end{tabular}

IIIb complications, 4 underwent a laparotomy and 2 an endoscopic procedure ( $\downarrow$ Table 2 ). The 9 tube dislodgments occurred at a median of 5 days ( $0-28$ days) postoperatively. 5 patients had a new gastrostomy tube inserted bedside. In 3 patients, bedside reinsertion was not possible; of these, 2 had a new 1 inserted endoscopically while one had a laparotomy due to inadequate transillumination during the initial procedure. Lastly, 1 patient had a laparotomy due to clinical signs of peritonitis. In all, the T-fasteners appeared to be in place, without obvious signs of migration. The balloon was noted to have deflated in 2 of the 9 patients.

1 or more early complications occurred in $48 \%$ of the infants and in $49 \%$ of those older than 1 year $(P=0.95)$. In patients weighing less than $10 \mathrm{~kg}, 50 \%$ had early complications, compared to $47 \%$ of those weighing more than $10 \mathrm{~kg}(P=0.77)$. When comparing patients with and without neurologic impairment, $49 \%$ and $47 \%$ experienced early complications, respectively $(P=0.86)$.

When comparing the first half of the patients with the last half, no difference was found concerning the frequency of early postoperative complications ( $44 \%$ vs. $52 \%, P=0.45$ ). With regard to grade IIIb complications, 3 occurred in the first half of patients, and the remaining 3 in the last half.

\section{Long-term results}

After the first 30 postoperative days, 55 gastrostomy-related complications were recorded in $33(38 \%)$ patients. 35 grade I complications were noted and included granulation tissue $(n=$ $17)$, stoma infection ( $n=12)$, and migrated T-fasteners $(n=6)$. Grade II complications were stoma infection $(n=12)$ and migrated T-fasteners $(n=2)$. Lastly, 8 grade IIIb complications were recorded: migrated T-fasteners $(n=5)$, tube dislodgment $(n=$ 2 ), and stricture of the stoma channel necessitating revision ( $n$ $=1$ ).

Records of change of the initial gastrostomy tube to a lowprofile button were found in 43 patients (49\%). In 39 patients, the initial tube was changed to a low-profile button as planned after about 2 months. Due to leakage, 4 patients had the tube changed earlier than planned. In 42 of the 43 patients (98\%), the change was done without the need for sedation or general anesthesia. In 1 patient, a short general anesthesia was necessary.

\section{T-fastener related complications}

Early and late T-fastener related complications occurred in 9 (11\%) and 11 patients (13\%), respectively ( $\triangleright$ Table 3 ). All late T-fastener complications seemed related to suspected migration of the T-fasteners into the stomach or abdominal wall. The late T-fastener complications were diagnosed at a median time of 2 months (4 weeks - 17 months) postoperatively. In the 10 patients requiring intervention, this was performed at a median time of 4.5 months ( 1.3 months -3.5 years) after PEG-T insertion. Pain related to migrated T-fasteners necessitated removal in 7 patients: 4 under general anesthesia and 3 without. Furthermore, 1 patient underwent endoscopy under general anesthesia because the remaining T-fasteners in the stomach wall were suspected of causing pain. However, no T-fasteners were found during the procedure. In 1 patient, pain related to migrated T-fasteners disappeared spontaneously. Lastly, migrated T-fasteners were discovered accidentally in 2 patients, but no procedures were performed because these children were asymptomatic.

\section{Discussion}

The main and surprising findings from this study are that many patients experienced dislodgment of the balloon tube within the first month and that the T-fasteners of the PEG-T system caused considerable problems. Although the use of T-fasteners is becoming increasingly popular in pediatric patients needing a gastrostomy, very few studies have addressed problems with Tfasteners in this population. In 1 retrospective study of 92 pediatric patients undergoing laparoscopic assisted PEG-T, only 3 patients (3\%) experienced problems with the T-fasteners; 1 needed an endoscopic evaluation, and 2 had migrated T-fasteners removed under local anesthesia [8]. In contrast, 2 other studies including 201 [6] and 186 [7] children reported no Tfastener related complications. In adults, T-fasteners are often used, especially during insertion of a gastrostomy under radiologic surveillance. None of the 71 adult patients included in a 
- Table 3 Early and late complications specifically related to T-fasteners in pediatric patients undergoing insertion of a percutaneous endoscopic gastrostomy with T-fasteners.

\begin{tabular}{|c|c|c|}
\hline & Early T-fastener complications $(n=10)$ & Late T-fastener complications $(n=13)$ \\
\hline Grade I & 6 & 6 \\
\hline - Infection at site of external suture lock & 5 & \\
\hline - Detached external suture lock & 1 & \\
\hline - Migrated T-fastener causing pain & & 4 \\
\hline - Migrated T-fastener without pain & & 2 \\
\hline Grade II & 4 & 2 \\
\hline - Infection at site of external suture lock & 3 & \\
\hline - Pain from external suture lock & 1 & \\
\hline - Infection at site of migrated T-fastener & & 2 \\
\hline Grade IIIb & 0 & 5 \\
\hline - Migrated T-fastener causing pain & & 5 \\
\hline
\end{tabular}

retrospective study experienced problems with the T-fasteners [11]. In contrast, a prospective study of 48 adults reported complications attributed to T-fasteners, either pain or skin related problems, in 11 patients (23\%) [12].

It is largely unknown why and when some T-fasteners migrate through the stomach and abdominal wall and cause problems. Too tight locking of the sutures is considered to be a risk factor. Furthermore, it is possible that children are more prone to experience T-fastener migration than adults because they have thinner tissue layers. Apparently, the migration occurs early after PEG insertion. CT imaging in adult patients performed less than 4 weeks postoperatively, showed that $36 \%$ of T-fasteners had migrated into the anterior abdominal wall [11]. It is assumed that the T-fasteners may migrate into the abdominal wall during the initial procedure, especially during tract dilatation. Lastly, how soon the T-fastener sutures are cut after PEG insertion, may also influence the tendency for T-fastener migration.

In studies investigating gastropexy with T-fasteners, the sutures are cut between 5 days and 3-4 weeks postoperatively. Livingston et al. changed from cutting the T-fastener sutures 3-4 weeks postoperatively to 5 days after PEG insertion. After this change of practice, they did not report any T-fastener related complications [8]. Göthberg and Björnsson cut all sutures after 2 - 3 weeks, and they have not experienced any T-fastener related complications [6]. In our patients, the sutures were supposed to be cut 3-4 weeks postoperatively if not already detached. Unfortunately, data on when the sutures were actually cut were incompletely reported in the medical records. We also learned that some caregivers did not understand that the sutures were meant to be cut after 3-4 weeks. For instance, in 1 patient later needing surgical removal of a subcutaneous T-fastener, the external sutures were noted to be in place 5 weeks postoperatively. It is possible that the high complication rate related to T-fasteners in the present study may be partly explained by late removal of T-fastener sutures, but we cannot be sure about this since there are insufficient data on suture removal.

Early tube dislodgment is a feared complication after PEG insertion because the gastrocutaneous tract takes time to mature. Among our patients, $10 \%$ experienced early dislodgment. Compared to other reported series of PEG-P, this is a high number $[13,14]$. In 2 series of PEG-T [6,7], there was no report of any early dislodgment, while Livingston et al. reported this in 2 of 92 patients [8]. We have no apparent explanation for the high frequency of early dislodgment. To be able to insert a balloon gastrostomy tube, a wider opening in the abdominal wall is needed compared to PEG-P. This may increase the risk for tube dislodgment in the early postoperative period. Other possible explanations for early dislodgment are balloon malfunction, traction by the patient, balloon damage by the T-fasteners, and caregiver error (insertion of fluids in the balloon). One could also speculate that early T-fastener migration or removal may influence the tendency for dislodgment. In the patients who experienced early tube dislodgment in the present series, the T-fasteners appeared to be in place in all.

A major advantage of PEG-T is easy removal without the need for sedation or general anesthesia. This compares well with our experiences where $98 \%$ of the patients had their PEG$T$ removed in the outpatient clinic with no need for sedation or anesthesia. Another proposed benefit of T-fastener gastropexy is the fixation between the stomach and anterior abdominal wall making bedside reinsertion safe in case of early tube dislodgment. In our study, bedside reinsertion was possible in 5 of 9 patients, showing that T-fastener gastropexy cannot guarantee that a new gastrostomy tube may be inserted bedside.

Peristomal infections were common in our series and occurred in $25 \%$ of patients during the first 30 days after surgery. A lower infection rate after PEG-T insertion has been reported in 2 other pediatric series [6,7]. Different classifications and definitions of peristomal infections may explain these differing results. In line with previous studies on peristomal infections after 
PEG-T, the design of this study makes it impossible to support a hypothesis of a lower peristomal infection rate after PEG-T compared to PEG-P. A randomized controlled study would best investigate this.

In addition to peristomal infections, hypergranulation is a common complication after PEG. Fortunately, this tends to become less of a problem with time. In a series from Sweden including 201 pediatric patients, hypergranulation was reported in $23 \%$ of patients 6 months after PEG-T insertion [6]. Interestingly, in the same series, hypergranulation was experienced by only $5 \%$ in the PEG-P group. In a small series of 10 Korean pediatric patients getting PEG-T, 50\% developed granulation tissue [15]. In our series, hypergranulation was only reported in $4 \%$ of the patients. Since our data are based on a retrospective chart review, it is likely that the frequency of hypergranulation is underestimated. It has been hypothesized that the wide and deeper abdominal wall incision required to insert the balloon gastrostomy, may explain a higher frequency of hypergranulation after PEG-T.

Complications necessitating intervention under general anesthesia (grade IIIb complications) occurred in $7 \%$ of the patients in the present series. Among 92 pediatric patients getting a laparoscopy assisted PEG-T, grade IIIb complications were reported in $4 \%$ [7]. Fewer grade IIIb complications were found in a series of 201 PEG-T insertions, where only 2 children (1\%) experienced grade IIIb complications [6]. In the present study, 2 patients were suspected of having intraabdominal organ injury because of pneumoperitoneum and/or feces-looking secretion from the gastrostomy site together with impaired general condition. No damage was found during laparotomy; however, a large gastrostomy channel was noted in both patients. Pneumoperitoneum can be a normal finding after PEG insertion, but when found in a patient with signs of possible organ injury, such as fever or signs of peritonitis, further investigations are indicated [16]. Interestingly, in the series from Jacob et al., $7 \%$ of the patients were reported to have pneumoperitoneum [7]. In these patients, pneumoperitoneum did not warrant further surgery, but it is reasonable to assume that the patients in Jacob's series had some clinical symptoms since abdominal X-rays were taken.

When introducing a new technique, a learning curve with regard to operating time and complications is likely to occur. At our center, the mean duration of insertion of a PEG-T was 28 minutes, which is in line with previous literature. In the large Swedish series of 201 patients getting PEG-T, median time for PEG-T placement was 20 minutes [6]. We did not see a decrease in operating time or in the frequency of patients experiencing early complications in our study as reported in other series [7, $8]$, but this may partly be explained by the relatively high number of physicians involved. Jacob et al. found the PEG-T procedure to be technically challenging, and they experienced a significant learning curve with regard to both complications and operating time [7]. In line with this, Livingston et al. found that all postoperative complications with laparoscopy assisted PEG-T insertion occurred within the first 50 cases and within the first 40 cases for each surgeon [8]. These findings support keeping the number of physicians involved with the procedure to a minimum.

Limitations of this study include the retrospective nature of data collection. Thus, it is likely that some complications are missed since patients may seek help at their general practitioner or local hospital. It would also have strengthened the study and interpretation of results if we had compared PEG-P and PEG-T in the same institution. However, this was not possible since PEG-T was the preferred technique. Strengths include the use of a validated scoring system for complications, including all complications, not only those obviously related to the gastrostomy. The use of the Clavien-Dindo system makes it easier to compare results and avoids subjective terms such as minor and major complications. The Clavien-Dindo classification has been used in trials in adults for a long time and is also increasingly used in pediatric studies [17]. Since none of the previous studies on PEG-T have used a predefined and validated complication classification system, it is difficult to compare results between studies.

To conclude, our study on initial experience with the PEG-T technique shows that the T-fasteners caused considerable short- and long-term complications, and the rate of early tube dislodgment was high. We did not see a lower rate of peristomal infections, but our hypothesis that PEG-T facilitates easy removal of the gastrostomy tube was confirmed. After reviewing these results, we have changed routines so that only 1 team of 1 pediatric surgeon and 2 pediatric endoscopists, inserts the PEG-Ts. Furthermore, there is particular focus on not making the sutures too tight and to avoid traction while dilating the gastrostomy channel. Lastly, all patients are scheduled for removal of the sutures and evaluation of postoperative results 2 weeks postoperatively. Hopefully, this change of practice will reduce the complication rate. Based on our experience with introduction of PEG-T, we recommend that this procedure is performed by a small team until sufficient experience with the method is gained at each center.

\section{Competing interests}

None

References

[1] Braegger C, Decsi T, Dias JA et al. Practical approach to paediatric enteral nutrition: A comment by the ESPGHAN committee on nutrition. J Pediatr Gastroenterol Nutr 2010; 51: 110-122

[2] Fortunato JE, Troy AL, Cuffari $\mathrm{C}$ et al. Outcome after percutaneous endoscopic gastrostomy in children and young adults. J Pediatr Gastroenterol Nutr 2010; 50: $390-393$

[3] Yaseen M, Steele MI, Grunow JE. Nonendoscopic removal of percutaneous endoscopic gastrostomy tubes: morbidity and mortality in children. Gastrointest Endosc 1996; 44: 235 - 238

[4] Srinivasan R, Irvine T, Dalzell AM. Traction removal of percutaneous endoscopic gastrostomy devices in children. Dig Dis Sci 2010; 55: $2874-2877$ 
[5] Campoli PM, de Paula AA, Alves LG et al. Effect of the introducer technique compared with the pull technique on the peristomal infection rate in PEG: a meta-analysis. Gastrointest Endosc 2012; 75: 988 996

[6] Göthberg G, Björnsson S. One-step insertion of low-profile gastrostomy in pediatric patients vs. pull percutaneous endoscopic gastrostomy: Retrospective analysis of outcomes. JPEN J Parenter Enteral Nutr 2016; 40: $423-430$

[7] Jacob A, Delesalle D, Coopman S et al. Safety of the one-step percutaneous endoscopic gastrostomy button in children. J Pediatr 2015; 166: $1526-1528$

[8] Livingston MH, Pepe D, Jones S et al. Laparoscopic-assisted percutaneous endoscopic gastrostomy: Insertion of a skin-level device using a tear-away sheath. Can J Surg 2015; 58: $264-268$

[9] Berry JG, Poduri A, Bonkowsky JL et al. Trends in resource utilization by children with neurological impairment in the United States inpatient health care system: A repeat cross-sectional study. PLoS Med 2012; 9: e1001158

[10] Dindo D, Demartines N, Clavien P. Classification of surgical complications: A new proposal with evaluation in a cohort of 6336 patients and results of a survey. Ann Surg 2004; 240: 205 - 213
[11] Sydnor RH, Schriber SM, Kim CY. T-fastener migration after percutaneous gastropexy for transgastric enteral tube insertion. Gut Liver 2014; 8: 495-499

[12] Thornton FJ, Fotheringham T, Haslam PJ et al. Percutaneous radiologic gastrostomy with and without T-fastener gastropexy: A randomized comparison study. Cardiovasc Intervent Radiol 2002; 25: 467 471

[13] McSweeney ME, Kerr J, Jiang $\mathrm{H}$ et al. Risk factors for complications in infants and children with percutaneous endoscopic gastrostomy tubes. J Pediatr 2015; 166: $1514-1519$

[14] Avitsland TL, Kristensen C, Emblem R et al. Percutaneous endoscopic gastrostomy in children: A safe technique with major symptom relief and high parental satisfaction. J Pediatr Gastroenterol Nutr 2006; 43: $624-628$

[15] Gang M, Kim J. Short-term complications of percutaneous endoscopic gastrostomy according to the type of technique. Pediatr Gastroenterol Hepatol Nutr 2014; 17: 214-222

[16] Lee JY, Park KS. Pneumoperitoneum after percutaneous endoscopic gastrostomy: Does it have clinical significance? Intest Res 2015; 13 : 295-296

[17] Clavien PA, Barkun J, de Oliveira ML et al. The Clavien-Dindo classification of surgical complications: Five-year experience. Ann Surg 2009; 250: 187 - 196 